\title{
Ion Exchange Resins as Excipients for Drug Delivery: Issues for Reproducible Drug Loading
}

\author{
Daniel Zeiss*,1,2 and Annette Bauer-Brandl ${ }^{2}$ \\ ${ }^{1}$ Faculty of Health Sciences, Nord-Trondelag University College, 7800 Namsos, Norway \\ ${ }^{2}$ Department of Pharmaceutics \& Biopharmaceutics, Institute of Pharmacy, University of Tromso, Norway
}

\begin{abstract}
A method for repeatable loading of propranolol hydrochloride as a model drug onto the ion exchanger Amberlite ${ }^{\text {TM }}$ IRP69 was developed and different parameters for the loading process were evaluated due to their impact of the extent of loading. It was found that the extent of loading in equilibrium is widely independent of the experimental method used. However, a magnetic stirrer comminutes the ion exchanger particles, which hampers repeatable loading processes. For kinetic studies, it was found that the pharmacopoeia dissolution tester gives best repeatable results compared to other stirring devices (magnetic stirrer or shaking bath). Furthermore, evaluation of the loading profiles revealed complex kinetics, which can be best described by (at least) two independent processes. In addition, with different ratios between ion exchanger and propranolol, the degree of loading in the equilibrium follows an adsorption isotherm of the Langmuir type, and predicts loading capacity.
\end{abstract}

Keywords: Ion exchanger, cation exchanger, loading, drug delivery, propranolol, Amberlite, kinetics, Langmuir.

\section{INTRODUCTION}

Ion exchange materials were introduced into the field of pharmacy approximately fifty years ago. Since then, they have been used both as active pharmaceutical ingredients (API), like the anion exchanger cholestyramine for the treatment of hypercholesterolemia [1] and the cation exchanger polystyrene sulfonate for the treatment of hyperkalemia [2], and further as excipients in pharmaceutical preparations. The number of possible applications of ion exchangers as excipients, among them mainly cation exchangers, has increased during the past years. The applications range from the use as a tablet disintegrant (e.g. Polacrilin Potassium NF) [3] , drug stabilization agents (e.g.[4]), to the use in the field of drug-delivery technologies, including controlled release [5], iontophoretically assisted transdermal [6], ophthalmic [7], and taste-masking systems [8]. A selection of commercial examples of finished drug products on the market today are for example a morphine retard granulate as sustained release formulation (MST Retard Granulate, Mundipharma, [9]), a cough mixture containing dextromethorphan as a taste-masked formulation (NeoTussan Suspension, Novartis [9]), and an ophthalmic preparation containing betaxolol (Betoptic S, Alcon [10]). Despite the widespread use of drug-resin complexes in dosage forms, little attention has been paid in the literature on how to load the ion exchange material with the respective drug molecules. This subject should be a prerequisite for any dosage form development dealing with drug delivery systems comprising ion exchange materials. For the present work, propranolol hydrochloride was chosen as a model drug. The drug, used in the treatment of high blood pressure, need to be administered 2-3 times a day due to its half-life of approximately 4 hours. In order to ensure continuously constant blood levels and to increase

*Address correspondence to this author at the Høgskolen i Nord-Trøndelag, 7800 Namsos, Norway; Tel: +47-742-12380; Fax: +47-742-12301; E-mail: daniel.zeiss@hint.no patient compliance, a controlled-release dosage form is desirable providing ones-a-day administration. In addition, propranolol hydrochloride bound onto ion exchange material has already been investigated by a number of researchers [11-13], mainly in release tests. In the present study, we will describe a method for reproducible loading exemplified by loading propranolol- $\mathrm{H}^{+} \mathrm{Cl}^{-}$onto a pharmaceutically used cation exchanger, sodium polystyrene sulfonate, described in both the United States Pharmacopoeia (USP) [14] and the European Pharmacopoeia (Ph.Eur.) [15].

The schematic structure of sodium polystyrene sulfonate is represented by Fig. (1).

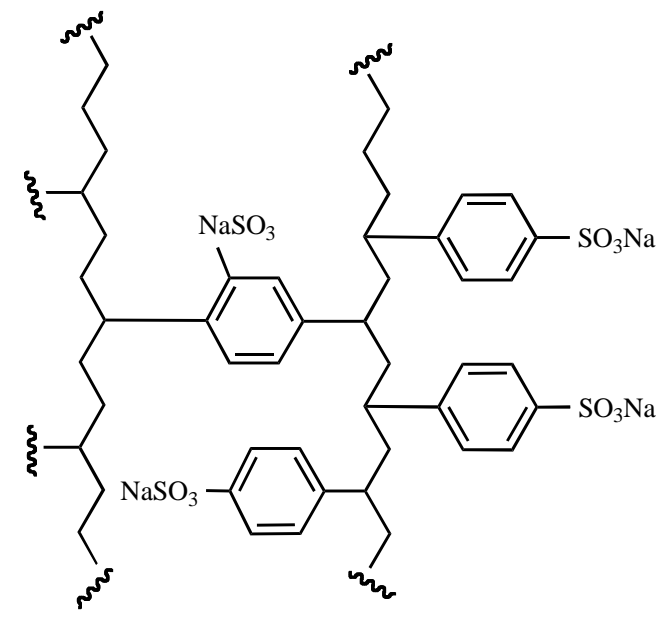

Fig. (1). Schematic molecular structure of sodium polystyrene sulfonate.

The resin is derived from a copolymer of styrene and divinylbenzene, which is then sulfonated. A commercially available material of pharmaceutical quality is Amberlite ${ }^{\mathrm{TM}}$ IRP69. The exchangeable cation in the commercialized form is sodium. Specifications are set to an exchange capacity of $2.8-3.4 \mathrm{mmol}$ of potassium ions per $\mathrm{g}$ of dry ion exchange 
material, and a content of sodium of $9.4-11 \%$ [16]. Amberlite ${ }^{\mathrm{TM}}$ IRP69 consists of polymer particles which are milled down to the requested particle size, the particle size distribution of which is described in the specifications as being 10$25 \%>75 \mu \mathrm{m}$, and maximum $1 \%>150 \mu \mathrm{m}[16]$.

The objective of the current study is to enable reproducible and optimum loading of Amberlite ${ }^{\mathrm{TM}}$ IRP69 with propranolol hydrochloride, in regard to both the extent and the kinetics of the loading. Furthermore, the kinetics of the loading process and of the ratio between free drug and drug bound onto the ion exchanger in the equilibrium is described.

\section{MATERIALS AND METHODS}

Amberlite ${ }^{\mathrm{TM}}$ IRP 69 (sodium polystyrene sulfonate USP; Lot 6210TD12) was obtained as a generous gift from Rohm and Haas France S.A.S. Chauny, France. ( \pm )-Propranolol hydrochloride (batch 1H106/1 and 5E045/1) was purchased from NMD AS, Oslo, Norway. All the substances were used as received. Distilled water was used as a solvent and dispersion medium.

To estimate the moisture content, electronic moisture analyzer Sartorius MA40 (Satorius, Göttingen, Germany) was used at $105^{\circ} \mathrm{C}$ for samples of approx. $2.5 \mathrm{~g}$ until constant.

For the electron micrographs, samples of dry Amberlite ${ }^{\mathrm{TM}}$ material were fixed to sticky tape, and sputtered with gold under vacuum. Bars in the micrographs indicate magnification.

Particle size analysis was done using an optical particle counter (AccuSizer ${ }^{\mathrm{TM}} 780$ Optical Particle Sizer, PSS Nicomp Particle Sizing Systems, Santa Barbara, California, USA), which estimates the total number of particles with a diameter between $1 \mu \mathrm{m}$ and $1000 \mu \mathrm{m}$ with a light-absorption detector and distributes it into classes. For measurements, the Amberlite $^{\mathrm{TM}}$ dispersion was diluted stepwise 1:1 using particle free water until the particle size distribution was unaltered by further dilution. This procedure ensures that single particles are detected in the flow line.

For the drug binding tests, a certain amount of ion exchange material was dispersed in distilled water in $250 \mathrm{~mL}$ bottles with screw cap. The drug solutions were added after the ion exchange material had swollen in the dispersion medium. The studies were carried out on a shaking water bath (Type 1086, GFL, Burgwedel, Germany), at $37^{\circ} \mathrm{C}$, or, alternatively, on a magnetic stirrer, at room temperature or at $37^{\circ} \mathrm{C}$. The size of the stirrer bar as well as the stirring speed were chosen to keep all the particles dispersed, while the frequency of the shaking water bath was chosen to be 100 $\min ^{-1}$ (maximum). After predetermined time intervals of either 24 hours or 48 hours, samples of $5 \mathrm{~mL}$ were taken with a syringe, and filtered through a $0.2 \mu \mathrm{m}$ membrane filter in order to remove the ion exchange particles. The free drug in the remaining solution was analyzed by UV spectroscopy.

Further binding tests were carried out in an apparatus 2 for drug dissolution tests [14] (Sotax AT 7 smart, Sotax AG, Basel, Switzerland) at $150 \mathrm{rpm}$ ensuring a homogeneous distribution of the ion exchange particles, at $37^{\circ} \mathrm{C}$, with a 10 fold up-scale of the reactants compared to the above mentioned experiments. Samples of $5 \mathrm{~mL}$ each were taken at several time points using syringes, and immediately filtered through $0.2 \mu \mathrm{m}$ filters.

Quantification of all samples was done by UV spectroscopy using a plate reader (Spectramax 190, Molecular Devices Corp., Sunnyvale, CA, USA) at $290 \mathrm{~nm}$ using standard curves.

For the Langmuir experiments, $1 \mathrm{~g}$ of ion exchange material Amberlite ${ }^{\mathrm{TM}}$ IRP 69 was dispersed in distilled water. Different volumes $(20 \mathrm{~mL}-150 \mathrm{~mL})$ of a $0.1 \mathrm{~mol} / \mathrm{L}$ propranolol hydrochloride aqueous solution were added and made up to the same total volume (dispersion medium + drug solution) of $1120 \mathrm{ml}$. The dispersions were kept at $37^{\circ} \mathrm{C}$ and stirred in a Sotax ${ }^{\text {TM }}$-dissolution-tester at 150rpm (Sotax AT 7 smart, Sotax AG, Basel, Switzerland). 48 hours after adding the respective drug solution to the dispersions, samples were taken with a syringe, filtered and quantified as described above. Amount of drug bound to the ion exchanger was calculated. Each experiment was carried out once.

\section{RESULTS AND DISCUSSION}

Amberlite $^{\mathrm{TM}}$ IRP 69 is a pharmaceutically used form of the sodium polystyrene sulfonate, which is available in the form of a milled powder. Its surface structure and an impression of the size distribution of the particles are depicted by electron micrographs in Fig. (2).
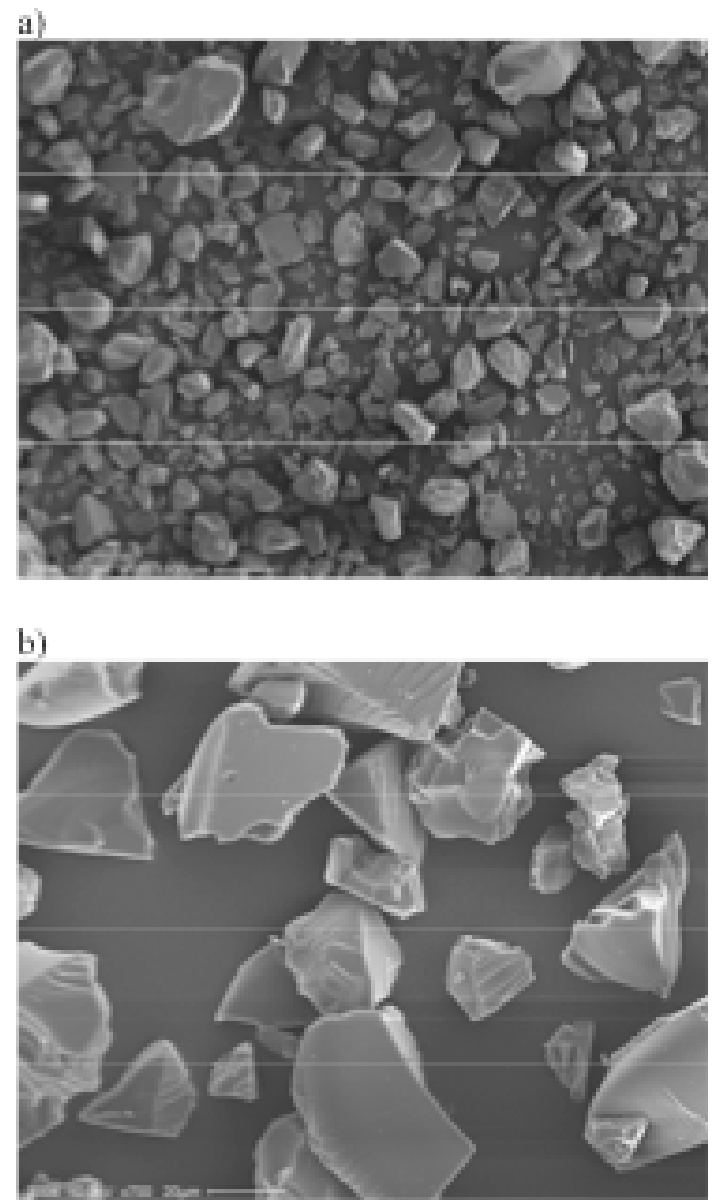

Fig. (2). a-b: SEM micrographs of Amberlite ${ }^{\mathrm{TM}}$ IRP69 dry powder; bars indicate magnification.

Since Amberlite ${ }^{\mathrm{TM}}$ IRP 69 is the salt of a strong acid and a strong base, its ion exchange ability is virtually independ- 
ent of $\mathrm{pH}$ [16]. No buffer was used in the present studies in order not to change ionic strength and hence equilibrium. It has been found that for the current system Amberlite ${ }^{\mathrm{TM}}$ IRP 69 / Propranolol $\mathrm{HCl}$, the $\mathrm{pH}$ value in the drug solution is 6 and does not change - within experimental error - during the course of the experiments (data not shown). As the $\mathrm{pK}_{\mathrm{A}}$ value of $\mathrm{PropH}^{+}$is 9.45 [17], it is concluded that the drug persists mainly in its ionic form during experiments, and that the $\mathrm{pH}$ would not need special attention.

\section{Moisture Content}

Exchange capacity is often related to the mass of dry material. The specifications of Amberlite ${ }^{\mathrm{TM}}$ IRP 69 reveal maximum water content of $10 \%$ [16] for the commercialized product, measured by drying in hot air. Values of loss on drying have been measured using an IR-balance, and they are in average $8.85 \% \pm 0.04 \%(\mathrm{n}=5)$, which complies with the mentioned specifications. Amberlite ${ }^{\mathrm{TM}}$ IRP 69 is a freeflowing powder. However, it cannot be excluded that under these conditions, the relative instability of the dry resin against decrease of the degree of sulfonization and / or of cross-linkage at elevated temperatures compared to the resin under moist conditions [18] makes normalizing the actual moisture content difficult. Therefore, the material used throughout the present study was kept in a tightly closed container, and the numbers given represent the mass of moist powder in all cases.

\section{Swelling}

The degree of swelling depends not only on the degree of cross-linking, on the degree of sulfonization and on other chemical properties of the resin, but also on temperature and on the type and concentration of ions in the solution (ionic strength). Furthermore, if the material is left to swell in a salt solution (e.g. drug solution), it is supposed that - as a first step - it would preferably take up water but not the drug ions, because the overall ion concentration is high within the particles. Therefore, the concentration of the drug solution added would initially increase. Dependent on the concentration of the added drug solution, the degree of swelling would be different, and it is assumed that this would affect the ex- change kinetics. In order to minimize this phenomenon, we have chosen to add the drug solution after leaving the particles swell in pure water for at least 4 hours. By this procedure, the starting point for all the dispersions is set to the same conditions (swollen particles), and the drug is added in the form of an aqueous solution at this point.

\section{Loading Procedure}

Little has been published on how to agitate the dispersions in drug loading experiments when reproducible kinetics is desired. Examples of methods that have been published for loading of Amberlite ${ }^{\mathrm{TM}} \mathrm{IRP} 69$ with propranolol-HCl include: a) "agitated for $24 \mathrm{~h}$ " [19] b) "shaken in a horizontal shaker at room temperature for $48 \mathrm{~h}$ " [13] and c) "mixtures were left in a dark room at room temperature $\left(25^{\circ} \mathrm{C}\right)$ for $48 \mathrm{~h}$ and shaken periodically" [11].

Using a shaking water bath, which is a common method used for loading ion exchangers $[11,13,18]$, the equilibrium of loading is far from being reached after 24 hours at $37^{\circ} \mathrm{C}$. It would rather take approximately 12 days to reach equilibrium (results from a preliminary study show an increase in amount drug bound from day 6 to day 12 of about $2.5 \%$ ). It was observed that part of the ion exchange material sediments in the flasks. In order to disperse the material evenly in the drug solution, a magnetic stirrer was used instead. The loading procedure was accelerated by stirring, but still 24 hours loading time is insufficient. It would rather take approximately $48 \mathrm{~h}$ to reach the degree of loading of about 700 $\mathrm{mg} / \mathrm{g}$ of ion exchanger at room temperature. This degree of loading is presumably the maximum amount that can be bound under the current conditions (ratio of drug, ion exchanger, and water). The huge difference in loading time between shaker and magnetic stirrer may indicate that the flow pattern of agitation is of significance for the kinetics of the ion exchange reaction (probably due to unstirred water layers on the surface of the particles). In contrast to the shaker, a magnetic stirrer yields a homogeneous dispersion of the ion exchange particles during the experiments, and enables shorter loading times probably due to the reduction in the width of the unstirred water layer on the surface of the particles.

Table 1. Loading of Samples Prepared at Different Temperatures and by Different Methods (Magnetic Stirring, Shaking Water Bath, and Dissolution Tester According to USP 29, Chapter 711, Apparatus 2)

\begin{tabular}{|c|c|c|c|c|c|c|}
\hline Amount ion exchanger (IE) / mg & $183 \pm 1$ & $183 \pm 1$ & $184 \pm 1$ & $\begin{array}{c}\text { Shaker } \\
183 \pm 1\end{array}$ & $1832 \pm 1$ & $1832 \pm 1$ \\
\hline Added volume drug solution / $\mathrm{mL}$ & $5.0 \pm 0.005$ & $5.0 \pm 0.005$ & $5.0 \pm 0.005$ & $5.0 \pm 0.005$ & $50.0 \pm 0.05$ & $50.0 \pm 0.05$ \\
\hline $\begin{array}{c}\text { Amount drug bound per mg IE / } \\
\mu \mathrm{g} / \mathrm{mg} \text { IE }(\text { mean } \pm \mathrm{sd})\end{array}$ & $659.84 \pm 16.42$ & $700.77 \pm 2.57$ & $698.87 \pm 1.89$ & $594.03 \pm 66.37$ & $701.25 \pm 0.84$ & $701.38 \pm 1.00$ \\
\hline Loading time / $\mathrm{h}$ & 24 & 48 & 48 & 48 & 24 & 48 \\
\hline Loading temperature $/{ }^{\circ} \mathrm{C}$ & 25 & 25 & $37.0 \pm 0.5$ & $37.0 \pm 0.5$ & $37.0 \pm 0.5$ & $37.0 \pm 0.5$ \\
\hline
\end{tabular}


Table 2. Preliminary Study for the Measurement of the Repeatability of the Loading of Propranolol Hydrochloride on the Ion Exchanger Amberlite IRP 69 with Five Replicates (Loading Time 24 Hours; Loading Temperature $25{ }^{\circ} \mathrm{C}$; Magnetic Stirrer Bench)

\begin{tabular}{|c|c|c|c|c|c|}
\hline & \multicolumn{5}{|c|}{ Replicate } \\
\hline Volume dispersion medium / mL & 100 & 100 & 100 & 100 & 100 \\
\hline Added volume drug solution / $\mathrm{mL}$ & 10 & 10 & 10 & 10 & 10 \\
\hline Amount drug bound per mg IE / $\mu \mathrm{g} / \mathrm{mg} \mathrm{IE}$ & 739.45 & 707.15 & 886.94 & 751.27 & 751.27 \\
\hline
\end{tabular}

Table 3. Particle Size Distribution by Number for Amberlite ${ }^{\mathrm{TM}}$ IRP 69 - Particles Swollen in Water; Before and after Stirring on a Magnetic Stirrer for 96 hours, as Measured by Optical Particle Counter

\begin{tabular}{|c|c|c|}
\hline & Untreated & After 96 hours stirring \\
\hline \hline Mode of particle size $/ \mu \mathrm{m}$ & 10.55 & 8.97 \\
\hline Mean \pm std.dev. & $2.21 \pm 0.155$ & $1.91 \pm 0.274$ \\
\hline Number of replicates & 6 & 5 \\
\hline
\end{tabular}

However, a preliminary experiment on the repeatability of the loading process shows that in parallel experiments on a magnetic stirrer bench (IKA RO 5 P, IKA Labortechnik, Staufen, Germany), assuring the same stirrer speed in all replicates, the data for the loading process were still not found sufficiently repeatable with a coefficient of variance of $>9 \%$ (Table 2) between replicates after 24 hours. Even the removal of one of the replicates considered as a potential outlier or failed experiment (replicate 3) would not yield a satisfying result with respect to the repeatability of the method (coefficient of variance 2.8\%).

In order to evaluate the reason for the limited repeatability, the effect of magnetic stirring on resin particles was studied. Particle size measurements in pure distilled water were carried out using a particle counter, covering a measuring range between $1 \mu \mathrm{m}$ and $1000 \mu \mathrm{m}$. A typical plot of the particle size distribution by number (expressed in \%) is shown in Fig. (3), the average of some characteristic descriptors of the distribution of 5 to 6 replicates is summarized in Table 3.

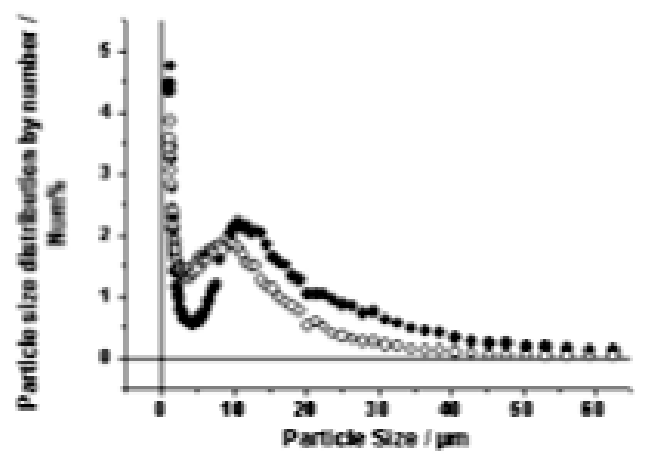

Fig. (3). Particle size distribution of Amberlite ${ }^{\mathrm{TM}}$ IRP69 swollen in distilled water before and after stirring on a magnetic stirrer for 96 h. Distribution by number; solid symbols: particles before treatment; open symbols: particles after treatment.
Particle size distributions comply with the specifications of Amberlite ${ }^{\mathrm{TM}}$ (see above): all the particles are below 60 $\mu \mathrm{m}$, with a large (number-based) fraction in the range of 1$10 \mu \mathrm{m}$ (see also Fig. $\mathbf{2 b}$ of the dry material for comparison).

Fig. (3) also shows the particle size distribution for a sample after stirring by a magnetic stirrer. The frequency of the larger particles decreases throughout, which means that the largest particles comminute towards a certain smallest favorable particle size.

This fact is regarded the reason for the surprisingly large deviations for replicates of loading on a magnetic stirrer: The degree of reduction in particle size by the magnetic stirrer, even at the same stirring speed and same flasks / stirrer bars, is prone to random effects caused by a non-laminar flow pattern. However, the findings indicate that particle size does not affect the degree of loading in equilibrium which is in agreement with former findings [12].

Using a overhead stirrer with defined flow pattern, as is introduced by the dissolution apparatus of USP [14] and Ph.Eur. [20], a homogeneous dispersion is yielded as well. The same degree of loading of approximately $700 \mathrm{mg} / \mathrm{g}$ of ion exchanger was reached (Table 1) within approx. 5 hours (Fig. 4), and stayed unchanged thereafter (equilibrium). Furthermore, in the dissolution tester, the reproducibility of the drug loading during the entire time of the process is shown in Fig. (4) for four replicates. The standard deviations are given as error bars, and they are lower than $5 \%$ throughout, after 30 minutes they are even lower than $1 \%$.

The repeatability of the degree of loading in the equilibrium is very good (below $0.5 \%$ ) for all methods. Given a molecular weight of 260.3 for the propranolol-cation, and $8.85 \%$ of water content for the ion exchanger, the binding capacity calculates to 2.95 meq per g dry material, which is in good agreement with the exchange capacity for potassium ions of about 2.8-3.4 meq per g dry ion exchange material. 


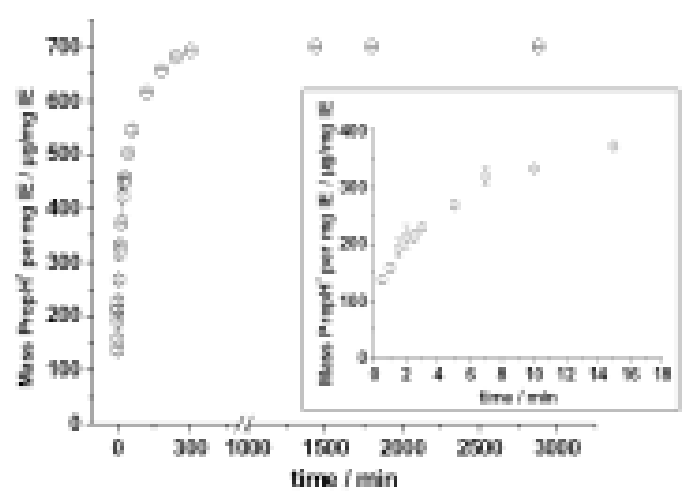

Fig. (4). Kinetics of loading of Propranolol-HCl onto Amberlite ${ }^{\mathrm{TM}}$ IRP 69 particles at $37^{\circ} \mathrm{C}$ in a dissolution tester. Mean and std.dev., $\mathrm{n}=4 ; 1.832 \mathrm{~g}$ ion exchanger, total volume $1120 \mathrm{ml} ; 0.1 \mathrm{~mol} / \mathrm{L}$ propranolol-HCl. In the box: Enlarged detail of the first 15 minutes.

Table 1 further shows that the same degree of loading (within experimental error) is reached independently whether the temperature is $25^{\circ} \mathrm{C}$ or $37^{\circ} \mathrm{C}$. This finding is in good agreement with results earlier reported [21], where reaction enthalpy and free Gibbs energy were measured calorimetrically at $37^{\circ} \mathrm{C}$, as to be $\Delta \mathrm{H}=-15.7 \mathrm{~kJ} \mathrm{~mol}^{-1}$, and $\Delta \mathrm{G}=-13.5$ $\mathrm{kJ} \mathrm{mol}^{-1}$. The entropy term therefore is $2.2 \mathrm{~kJ} \mathrm{~mol}^{-1}$ at this temperature, and disregarding any heat capacity changes with temperature, the Gibbs energy would change by less than $0.1 \mathrm{~kJ} / \mathrm{mol}$ by the temperature, which is within experimental error of the method [21].

In the present case, mass equilibrium constants for the reaction derived from equilibrium state concentrations are not calculated, because activities need to be regarded. In the resin, activities of ions are expected to be much lower than in the surrounding diluted solutions, they are widely unknown, and difficult to estimate.

\section{Evaluation of Loading Data}

It is generally acknowledged that the mechanism of loading of ion exchange materials batch-wise to equilibrium can be schematically divided into three main steps: film diffusion of exchanging ions (through the unstirred water layer on the surface of the particles), bulk diffusion within the particles, and the actual chemical exchange reaction. Since the chemical exchange reaction is assumed to be very fast compared to the two diffusion processes, the former process is not supposed to be rate determining. Some work has already been done in order to elucidate which of the two diffusion processes mentioned above is rate-determining. Helfferich developed an equation for an estimation, the so-called Helfferich number [22]. However, the mentioned equation as well as theoretical kinetic models are based on the use of ion exchange particles of a defined geometric shape and defined radius, respectively. For irregular-shaped particles, like Amberlite $^{\text {TM }}$ IRP 69 , the use of these models may lead to an increased uncertainty due to an inaccurate assumption of particle sizes, and reduced goodness of fit to the experimental data. In the present work, an attempt is made to estimate the rate-determining step in the investigated system from the loading kinetic, which is independent of shape of particles.

Fig. (4) shows the loading of Amberlite ${ }^{\mathrm{TM}}$ IRP69 with propranolol- $\mathrm{HCl}$ in water, with small standard deviations for
4 replicates, and the velocity of the reaction is therefore regarded repeatable and suitable for kinetic evaluation. The equilibrium is reached after approx. 5 hours, and the fact that it would take much longer in a shaking water bath (Table 1) indicates that film diffusion rather than particle diffusion is the rate-determining step in those experiments where the agitation is slow. For film diffusion, the initial exchange velocity would be linear with time in the beginning, which means in this case approx. during the first minute(s). It has been practically impossible to collect more samples during the first minutes with suitable accuracy, than are shown in the detail plot of Fig. (4). Nevertheless, it can be concluded that there is no linear initial phase, and it is further concluded that film diffusion is not the rate-limiting step under the chosen conditions (150 rpm in dissolution tester). Slower stirrer speeds could possibly lead to significant impact of the film diffusion, but seem not applicable for kinetic studies since the resin would tend to sediment, which in turn would hamper reproducible flow patterns and diffusion properties around the particles.

In order to further evaluate the kinetics of binding with respect to bulk (particle) diffusion, the equation originally applied to ion exchange reactions by Boyd, Adamson and Myers [23] (equation 1) was used.

$F=\frac{M_{t}}{M_{\infty}}=1-\frac{1}{\pi^{2}} \sum_{n=1}^{\infty} \frac{e^{-n^{2} B t}}{n^{2}}$

Equation (1)

where $\mathrm{F}$ is the fraction absorbed at time $\mathrm{t}, \mathrm{M}$ is the mass absorbed at time $t$ or respectively at infinite time (equilibrium), and

$B=\pi^{2} D^{i} / r^{2}$

Equation (2)

where $D^{i}$ is the effective diffusion coefficient of the two exchanging ions inside the resin.

The fraction of the drug bound to the ion exchanger plotted semilogarithmic vs. time is shown in Fig. (6) (open symbols). In contrast to similar studies with other ions [24] (exchanging $\mathrm{H}^{+}$vs. $\mathrm{Na}^{+}$), here it was found that the function is not linear. However, an analogous study to the present one describing the exchange of $\mathrm{Na}^{+}$vs. propranolol- $\mathrm{H}^{+}$[12], also revealed a non-continuous function for the loading, the reason for which is not explained in the paper. From the present findings it is concluded that the process studied cannot be described by a simple first order process, which means that the rate-determining step is not just particle diffusion with constant effective diffusion coefficients, neither for $\mathrm{Na}^{+}$nor for propranolol- $\mathrm{H}^{+}$ions.

In order to investigate whether the non-linearity is related to a more complex kinetic or the irregularity of the ion exchange particles, the models of Peppas and coworkers were applied on the current system. These models are originally developed to describe the release of solutes from polymeric devices by a Fickian or a non-Fickian mechanism. The release from devices of different shapes (slabs, spheres, cylinders or discs) was examined, and mathematical models based on Fick's second law posted. Assuming that both the loading process of drugs onto the ion exchange material and the release process from the ion exchange material follow the same mechanism principle, Peppas equation [25] was applied: 


$$
\frac{M_{t}}{M_{\infty}}=k t^{n}
$$

where $\mathrm{M}_{\mathrm{t}} / \mathrm{M}_{\infty}$ is the fraction of drug bound to the ion exchange material, $\mathrm{t}$ is the loading time, $\mathrm{k}$ is a constant containing information about the interaction between macromolecular network system and the drug, and $\mathrm{n}$ is the diffusional exponent, which is indicative of the transport mechanism.

The plot applied to the present system looks as shown in Figs. (5a and 5b). Fig. (5a) shows the fit for diffusion through one-dimensional devices like slabs, where $n=0.5$, and the equation is formally equivalent to the well-known Higuchi equation (square root of time plot) [26]. Due to the dependency of the parameter $\mathrm{n}$ on the geometry of the particles, Fig. (5b) shows the plot for (idealized) spherical particles, where $\mathrm{n}=0.43$.

a)

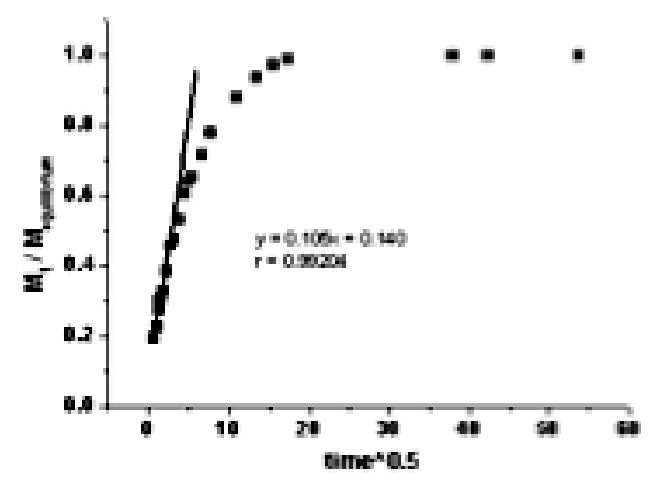

b)

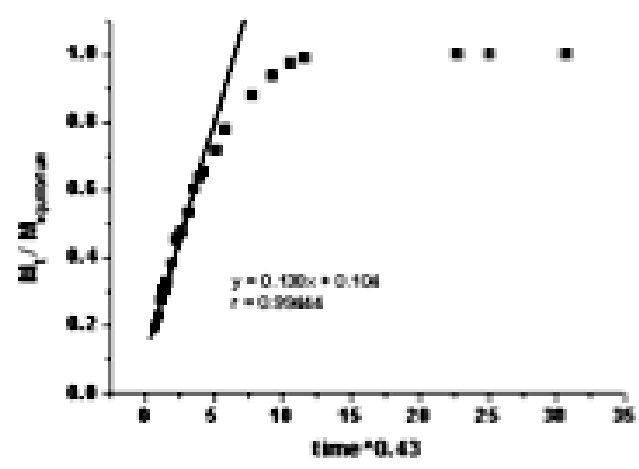

Fig. (5). Peppas plot (equation 3) same data as in Fig. 4; a) $n=0.5$; b) $n=0.43$.

As can be seen from the figures, in both cases the respective fits up to a limit of 0.6 fraction bound - as proposed by Peppas - seem to look fine, with correlation coefficients for both approaches to be approximately the same. According to the model, $\mathrm{n}$ is supposed to be $0.5,0.45$ or 0.43 for Fickian diffusion from slabs, cylinders and spheres, respectively, and even higher for non-Fickian transport. For the current system of drug and ion exchange material however, the fit reached an optimum for $\mathrm{n}=0.31$ (comparison of all linear fits between $\mathrm{n}=0.5$ and $\mathrm{n}=0.2 ; \mathrm{n}=0.31$ gave the best fit with a correlation coefficient $r=0.99613$ ), which is far below the theoretical lowest value for the spherical particles. Due to the observed particle shape of the ion exchange material, $\mathrm{n}$ should be between 0.5 and 0.43 for Fickian diffusion, or even higher for non-Fickian transport. The results give rise to the conclusion that the non-linearity behavior of the semilogarithmic plot of drug bound vs. time mentioned above is not related to the irregular particle shape, but rather seems to be related to the loading mechanism itself.

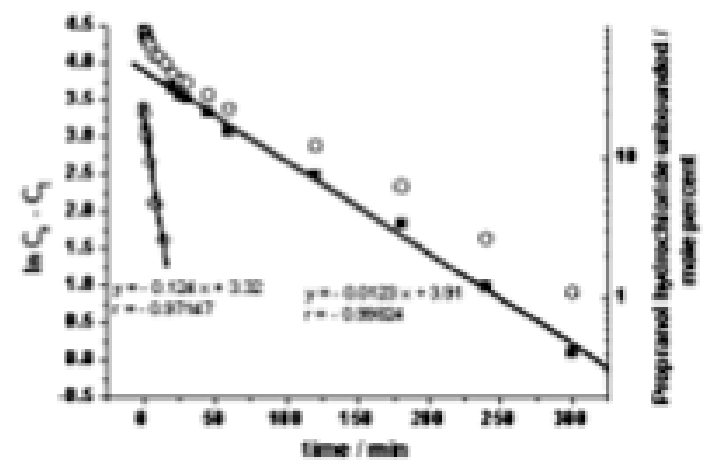

Fig. (6). Semilog Sigma-minus plot (open symbols) of loading of Propranolol-HCl onto Amberlite TM IRP 69, experimental details see Fig. (4). Solid symbols: Splitting into two independent firstorder processes.

For further investigation of the loading mechanism, the binding process was mathematically split up into two different parallel processes of first order kinetics, as shown by the solid symbols in Fig. (6). As can be seen from the figure, there is a factor of approximately 10 between the kinetic constants of the two parallel processes, of which the fastest dominates in the beginning, and the slowest takes over during later course of the exchange reaction. The points, particularly the ones describing the fastest process, seem not to fit to the model as good as in the Peppas model due to a lower correlation coefficient. However, considering the more complex mathematical procedures of the "splitting" method compared to the Peppas model, the experimental data fits quite well into the two first-order kinetic processes. Therefore, it is concluded that in this particular example, the loading of the ion exchanger is a complex process, which may include at least two different parallel mechanisms.

Using an equation coupling diffusion and relaxation (i.e. swelling) of the devices, also proposed by Peppas [27],

$\frac{M_{t}}{M_{\infty}}=k_{1} t^{m}+k_{2} t^{2 m}$

(Equation 4)

with $\mathrm{m}=0.43$ as is used for spheres, a better overall fit (up to a limit of approx. 0.8 fraction bound) results as depicted in Fig. (7).

The equation couples the diffusion step with a relaxation step for swelling objects as two simultaneous processes of each of its own kinetics. (Formally, this method is similar to the above-mentioned splitting into two first order kinetics). In the present case, Peppa's equation is used as formalism, since there is no substantial swelling of the particles within this experiment. In the present case, however, the changing of matrix diffusion properties may rather be related to the increasing load of the resin with large drug molecules. The fit to the equation is given in Fig. (7), as well as the calculated parameters, where the parameter for the "relaxation" is negative, meaning that the velocity of the reaction decreases as the resin gets "tighter" and "tighter" while more and more 


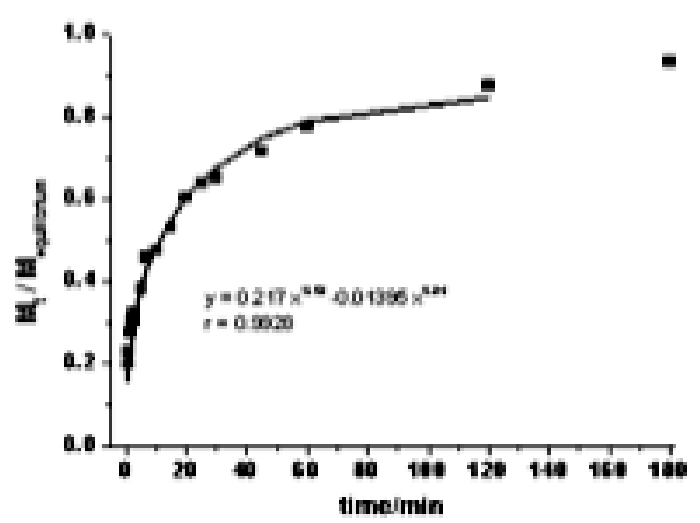

Fig. (7). Fit to equation 4; experimental details see Fig. (4).

propranolol- $\mathrm{H}^{+}$is bound. Calculating the ratio of the "tightening" effect over Fickian contribution by equation 5 :

$$
\frac{R}{F}=\frac{k_{2}}{k_{1}} t^{m}
$$

(Equation 5)

, one finds the impact of "tightening " to increase from $17 \%$ after 10 minutes, to $37 \%$ after $1 \mathrm{~h}$, and $50 \%$ after $2 \mathrm{~h}$. This indicates that the drug loaded into the matrix affects the kinetics of further loading. The mathematical description of this mechanism is valid up to approx. $80 \%$ of the loading. However, it does not provide sufficient prediction of the equilibrium degree of loading.

For this purpose, the ion exchanger may be regarded as a matrix with restricted number of binding sites. A Langmuir adsorption isotherm-based approach can then be used, similarly as has been done in [28-32], according to Equation 6:

$$
\frac{c}{y}=\frac{1+b * c}{y_{\max } * b}
$$

, with $c=$ equilibrium concentration, $y / y_{\max }=\Theta ; b=$ dimensionless constant.

By plotting c/y vs. c, a linear relationship is expected, as shown in Fig. (8), where the correlation is found to be excellent.

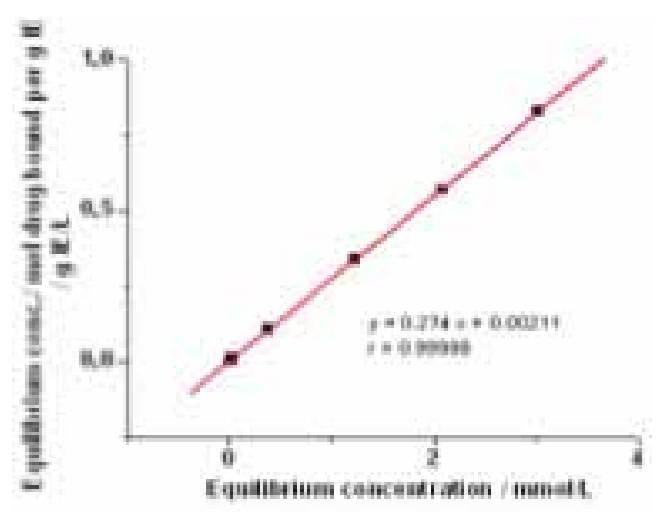

Fig. (8). Langmuir Plot Propranolol hydrochloride/Amberlite $\mathrm{T}^{\mathrm{TM}}$ IRP 69; Amount ion exchange-material 1.000g; concentration propranolol hydrochloride solution $0.1 \mathrm{~mol} / \mathrm{L}$; volume propranolol hydrochloride solution $20 \mathrm{~mL}-150 \mathrm{~mL}$; total volume $1120 \mathrm{~mL}$;

This would indicate that the mechanism of the Langmuir approach (i.e. a restricted number of binding sites and 1. order kinetics of both sorption and desorption in equilibrium) is valid in the present case, which in turn is consistent with suggestions for other ion-exchange drug systems, e.g. [28].

From the Langmuir plot (Fig. 8), the slope $1 / y_{\max }$ can be found, and a maximum loading $\left(\mathrm{y}_{\max }\right)$ of 3.65 meq per $\mathrm{g}$ ion exchange material calculated, corresponding to 4.00 meq per $\mathrm{g}$ dry ion exchanger. This value is significantly larger than the specifications of the ion exchange material for the exchange of potassium-ions. This fact may indicate that propranolol hydrochloride may not be able to interact with the ion exchange material by ion-ion forces, but also by other forces.

\section{CONCLUSIONS}

The present study shows that the degree of loading Amberlite $^{\text {TM }}$ IRP69 with propranolol hydrochloride to equilibrium is independent of particle size, and also independent of temperature in the interval $25^{\circ} \mathrm{C}$ to $37^{\circ} \mathrm{C}$. At $37^{\circ} \mathrm{C}$, equilibrium is reached after $5 \mathrm{~h}$ in the pharmacopoeia dissolution tester at $150 \mathrm{rpm}$. Under these conditions, film diffusion does not play a major role in the kinetics of the loading. The kinetics of the ion exchange reaction is complex. It may be described by a coupling of bulk diffusion and simultaneously "tightening" of the matrix, with increasing impact over time. A Langmuir adsorption isotherm reveals an extremely good data fit, and would allow for the prediction of the extent of drug loading for other drug solution / ion exchanger ratios, as well as the loading capacity.

\section{ACKNOWLEDGEMENTS}

The authors would like to thank Merete Skar, Dorothee Kohler, and Siri Forsdahl for technical help.

\section{REFERENCES}

[1] Brown, W. V. Am. J. Cardiol., 1990, 66, 11 A.

[2] Bernard, H. R.; Fletcher, J. C.; Humphreys, C. F. A. M. A. Arch. Surg., 1958, 77, 703.

[3] van Abbe, N. J.; Rees, J. T. J. Am. Pharm. Assoc. (1912-1977), 1958, 47, 487.

[4] Kankkunen, T.; Huupponen, I.; Lahtinen, K.; Sundell, M.; Ekman, K.; Kontturi, K.; Hirvonen, J. Eur. J. Pharm. Sci., 2002, 16, 273.

[5] Halder, A.; Sa, B. J. Microencapsulation, 2006, 23, 899.

[6] Jaskari, T.; Vuorio, M.; Kontturi, K.; Urtti, A.; Manzanares, J. A.; Hirvonen, J. J. Controlled Release, 2000, 67, 179.

[7] Singh, O. N.; Castillo, E. J. Sustained release formulations comprising a cation exchange resin and a combination of polymeric suspending agents. 2002-295284 7001615, 2006.

[8] Hargens Robert, D.; Jia, Y.; Slominski, G.; Vayalakkada, S. Tastemasked composition containing a cation exchanger. 2004-EP8394 2005013934, 2005.

[9] Rote Liste. Arzneimittelverzeichnis für Deutschland. http:www.rote-liste.de/online/index_html (accessed Feb. 28,2007)

[10] Felleskatalogen. Felleskatalog over Farmas øytiske Spesialpreparater Marketsført i Norge. http://www.felleskatalogen.no/ (accessed Mar. 1,2007)

[11] Akkaramongkolporn, P.; Terada, K.; Yonemochi, E. Drug Dev. Ind. Pharm., 2001, 27, 359.

[12] Burke, G. M.; Mendes, R. W.; Jambhekar, S. S. Drug Dev. Ind. Pharm., 1986, 12, 713.

[13] Sriwongjanya, M.; Bodmeier, R. Int. J. Pharm., 1997, 158, 29.

[14] The United States pharmacopeia, 29th ed.; United States Pharmacopeial Convention: Rockville, Md., 2006.

[15] Sodium Polystyrene Sulphonate. European Pharmacopoeia 5.6. http://online.pheur.org/entry.htm (accessed Mar. 8,2007)

[16] Rohm \& Haas website. Amberlite data sheet. http://www.rohmhaas.com/ionexchange/Pharmaceuticals/IRP69_do wnload.htm. (accessed Jan. 15,2007) 
[17] Martinez-Gomez, M. A.; Villanueva-Camanas, R. M.; Sagrado, S.; Medina-Hernandez, M. J. Electrophoresis, 2005, 26, 4116.

[18] Rieman, W.; Walton, H. F. Ion exchange in analytical chemistry; Pergamon: Oxford, 1970.

[19] Sriwongjanya, M.; Bodmeier, R. Eur. J. Pharm. Biopharm., 1998, 46,321 .

[20] Dissolution Test for Solid Dosage Forms. European Pharmacopoeia 5.6. http://online.pheur.org/entry.htm (accessed Mar. 8,2007)

[21] Zeiss, D.; Bauer-Brandl, A. J. Therm. Anal. Calorim., 2006, 83, 309.

[22] Helfferich, F. G. Ion exchange; McGraw-Hill: New York, 1962.

[23] Boyd, G. E.; Adamson, A. W.; Myers, L. S., Jr. J. Am. Chem. Soc., 1947, 69, 2836.
Reichenberg, D. J. Am. Chem. Soc., 1953, 75, 589.

Ritger, P. L.; Peppas, N. A. J. Controlled Release, 1987, 5, 23.

Higuchi, T. J. Pharm. Sci., 1961, 50, 874.

Peppas, N. A.; Sahlin, J. J. Int. J. Pharm., 1989, 57, 169.

Abdekhodaie, M. J.; Wu, X. Y. Biomaterials, 2006, 27, 3652.

Boudy, V.; Voute, N.; Pradeau, D.; Chaumeil, J. C. Int. J. Pharm., 2002, 239, 13.

[30] Boyd, G. E.; Schubert, J.; Adamson, A. W. J. Am. Chem. Soc., 1947, 69, 2818.

[31] Johns, W. H.; Bates, T. R. J. Pharm. Sci., 1969, 58, 179.

[32] Misak, N. Z. Colloids Surf., A, 1995, 97, 129. 